\title{
An experimental and numerical analysis of the fluid flow in a mechanically agitated vessel
}

\author{
Marek Jaszczur", , Anna Młynarczykowska ${ }^{1}$, Luana Demurtas ${ }^{1,2}$ \\ ${ }^{1}$ AGH University of Science and Technology, Faculty of Energy and Fuels, A. Mickiewicza 30, 30-059 Kraków, Poland \\ ${ }^{2}$ University of Cagliari, Department of Ingegneria Civile, Ambientale e Architettura, Via Marengo 2, 09123 Cagliari, Italy
}

\begin{abstract}
The mixing process is a widespread phenomenon, which plays an essential role among a large number of industrial processes. The effectiveness of mixing depends on the state of mixed phases, temperature, viscosity and density of liquids, mutual solubility of mixed fluids, type of stirrer, a what is the most critical - the shape of the impeller. In the present research, the objective is to analyse the process of the fluid flow in the mechanically agitated vessel with new impeller type. Velocity field values were determined using computer simulation and experimental particle image velocimetry method. The basis for the assessment of the intensity degree and efficiency of mixing was the analysis of velocity vectors distribution and power number. An experimental and numerical study was carried out for various stirred process parameters to determine optimal conditions for the mixing process.
\end{abstract}

\section{Introduction}

Stirred vessels are widely used not only in chemical processes but also in bioprocess, food industry and more recently in wastewater treatment and mineral processing, to accomplish homogenization, gas dispersion, solid suspension, heat transfer, etc. [1]. It also plays an essential role in selected processes as gas dispersion in liquids, the formation of suspensions and slow-sedimenting mixtures, prevention of sediment aggregation. In a large number of studies, researchers achieved a certain degree of awareness on basic development and mechanism of fluid mixing, above all, on single-phase flow. Nevertheless, there is still insufficient information on the behaviour of unbaffled stirred tanks which best fits in pharmaceutical processes, that require a high standard of cleanness, or in shear-sensitive cultures, that need relevant gas intake [2].

Furthermore, as for every industrial process, the main target has ever been to find an efficient way of mixing using as low power as possible. For this reason, several studies have been done to obtain different shapes of impellers that could achieve a good mixture quality with low axial velocity, low internal heat generation (important for vitamins, enzymes and in food production) and with as low as possible power consumption. Undeniably, the power used can be decreased improving the shape of the impeller. The study of T. Su et al. [3] compared the turbulent hydrodynamics of the liquid phase flow and mixing process using the Rushton impeller and a modified one, the covering-plate Rushton impeller. The analyses reached $18 \%$ of power decrease by modifying the Rushton impeller, which also managed to alter the local flow pattern and obtain more turbulence around it, eventually decreasing the mixing time required.
F. Scargiali et al. [4] analyse the influence of Reynolds and Froude numbers on power consumption characteristics in the case of unbaffled stirred tanks. Authors found that the most critical factor in decreasing power consumption and mixing quality is the shape of the impeller. Hence, many studies have been done to find the optimal shape for the various operating situation. T. Kumaresan and J. B. Joshi [5] worked using LDA measurements and Computational Fluid Dynamics (CFD) predictions with sliding mesh approach, to study the effect of impeller geometry on the fluid flow pattern and mixing time for a set of axial flow impellers (pitched blade turbines and hydrofoils). Authors obtain a very good agreement between experimental and predicted mixing time over a wide range of impellers geometries varying in a number of blades, blade angle, blade width and impeller diameter.

M. Basavarajappa et al. [6] investigate turbulent single-phase flow characteristics in lab-scale stirred tanks with different geometry using CFD simulations and comparing the Rushton turbine with the flotation impeller. $\mathrm{H}$. Ameur et al. [7] investigated a 3D numerical simulation to study the effects of blade curvature, diameter, blade number and Reynolds number, observing that the curved blades are the most efficient to reduce the power consumption [8-10].

In the past, mechanically agitated vessel design was restrained to the mere empirical experiment, as a result of lack in computational fluid dynamics reliability and inadequacy of computer power and memory. At present, advanced computer technology and complex mathematical models allow researchers to rely on numerical analysis in order to predict the hydrodynamics of more and more complex systems, replacing the time-

* Corresponding author: jaszczur@agh.edu.pl 
consuming and expensive real experiments. On the other hand, due to the complexity of flow phenomenon, experimental analysis is still required [11] as a final step of analysis or optimisation process. A. Delafosse et al. [12] performed a research study on the behaviour of flow rates between two adjacent compartments using CFD modelling. The results show that the CFD-based compartment model was able to reproduce with good accuracy the spatial distribution of concentrations during the mixing process, without any adjustable parameters. Along with CFD simulation and PIV (Particle Image Velocimetry), Large Eddy Simulation (LES) and Direct numerical Simulations (DNS) become a very promising technique $[13,14]$ to solve a problem at spatial and temporal scales explicitly. Indeed, in the case of flows with unsteady boundary conditions, such as the one in a stirred tank, LES method can be much better suitable to resolve the phenomena related to the unsteady stirring conditions than others methods [14]. S. Malika et al. [15] used LES method to produce a low-cost computational model, without losing a good agreement between simulation and PIV results. Authors showed that to improve CFD results it is essential to perform the grid numerical solution dependence study, and test the optimal size of the inner rotating fluid zone for multiple reference frame (MRF) models.

A few studies have been done to develop the CFD model and to characterise the hydrodynamics inside the stirred tank. Those analyses are ultimately useful for the optimal design of tanks, and there is still the necessity to extend current knowledge about fluid physics in a stirred vessel. Although H. Patil et al. [16] developed a CFD model for a stirred tank to optimise the dimensions of the inner rotating fluid zone using MRF model and to consider the optimal zone where simulation results for velocity predictions were found to be in reasonable agreement with literature data available. Authors investigated the efficiency of optimal MRF zone by comparing the power number predictions at various Reynolds numbers with the experimental results of J. H Rushton et al. [17].

Many researchers have made assessments of different turbulent models. The analyses presented by H. Singh et al. [18] compared different turbulence models, including $k-\varepsilon$, exposing the accuracy of prediction for the mean axial and tangential velocities and using all analysed models. However, results failed to predict the decay of mean radial velocity away from the impeller. They are noticing that the $k-\varepsilon$ model predicts the random and periodic components of the kinetic energy of fluctuating motion poorly in the vicinity of the impeller. The $k-\varepsilon$ model has also been compared to the RSTM and LES in the work of B. N. Murthy et al. [19]. Authors performed LDA measurement as well as CFD simulation for the flow generated by various impellers. Like for the study of $\mathrm{H}$. Singh, authors managed to find a turbulence model that well fits the experimental results whereas, the standard $k-$ $\varepsilon$ model underpredict the turbulent kinetic energy profile in the impeller region and failed to simulate the mean flow associated with the strong swirl. Depending on what the mixing process has to carry out, it is appropriate to select different configurations. However, there is a poor choice of impellers suitable for a wide range of various situations as well as operating conditions. For this reason, the aim of the present work is to provide and analyse a new impeller type that has a good efficiency and low power consumption and at the same time are suitable for mixing a wide variety of fluids creating a satisfying quality product. This paper focus on mixing optimisation using the power number, mean axial velocity and mean radial velocity, by means of the standard $k-\varepsilon$ turbulent model and experimental measurement using Particle Image Velocimetry technique.

\section{Analysis and modelling}

The mechanically agitated vessel configuration is presented in Figure 1, and the dimension used are shown in Table 1. For presented results, water was used as a working fluid with a variable viscosity for different temperatures. The distance between the impeller and the bottom of the tank $h$ was constant and equal to $8 \mathrm{~cm}$.

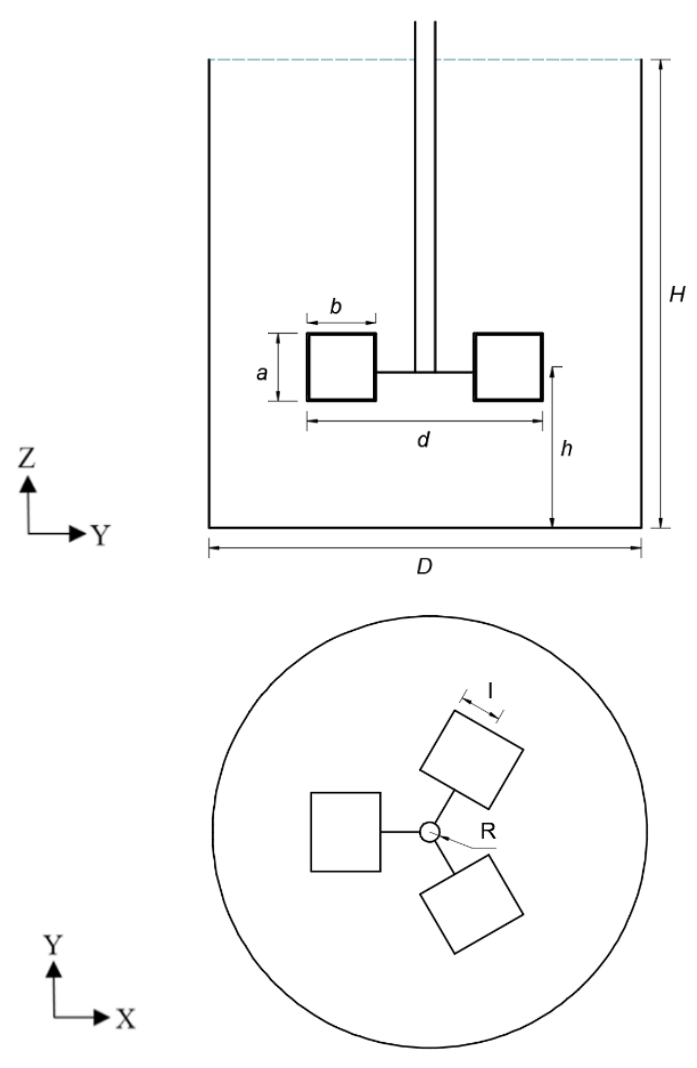

Fig. 1. Dimensions of turbine stirred tank.

Table 1. Dimensions of the stirred tank.

\begin{tabular}{ccccccc}
\hline $\begin{array}{c}\mathrm{H} \\
\mathrm{cm}\end{array}$ & $\begin{array}{c}\mathrm{h} \\
\mathrm{cm}\end{array}$ & $\begin{array}{c}\mathrm{D} \\
\mathrm{cm}\end{array}$ & $\begin{array}{c}\mathrm{d} \\
\mathrm{cm}\end{array}$ & $\begin{array}{c}\mathrm{a} \\
\mathrm{cm}\end{array}$ & $\begin{array}{c}\mathrm{b} \\
\mathrm{cm}\end{array}$ & $\begin{array}{c}\mathrm{l} \\
\mathrm{cm}\end{array}$ \\
\hline 22 & 8 & 22 & 13 & 4 & 3 & 1.9 \\
\hline
\end{tabular}

The steady-state of flow behaviour inside the stirred tank is described by governing equations for mass and momentum conservation. The continuity equation, for a threedimensional analysis, is as follows: 


$$
\frac{\partial u_{1}}{\partial x_{i}}+\frac{\partial u_{2}}{\partial x_{j}}+\frac{\partial u_{3}}{\partial x_{k}}=0
$$

For momentum conservation, assuming incompressible fluid, the Navier-Stokes equations are given by:

$$
\rho \frac{D u_{i}}{D t}=\frac{\partial u_{i}}{\partial x}+\mu\left(\frac{\partial^{2} u_{i}}{\partial x_{i}^{2}}+\frac{\partial^{2} u_{i}}{\partial x_{j}^{2}}+\frac{\partial^{2} u_{i}}{\partial x_{k}^{2}}\right), i=1,2,3
$$

where $u_{i}$, is the velocity component respectively, in the $i$, $j$ and $k$ direction. The set of eq.(1)-(2) is averaged using the Reynolds decompositions which allow separating mean and fluctuating components of an instantaneous quantity. The equations (3) and (4) are indeed the corresponding turbulence equations:

$$
\begin{gathered}
\frac{\partial \rho}{\partial t}+\frac{\partial\left(\rho u_{i}\right)}{\partial x}=0 \\
\frac{\partial\left(\rho u_{i}\right)}{\partial t}+\frac{\partial\left(\rho u_{i} u_{j}\right)}{\partial x_{j}}= \\
=-\frac{\partial p}{\partial x_{i}}+\frac{\partial}{\partial x_{j}}\left[\mu\left(\frac{\partial u_{i}}{\partial x_{j}}+\frac{\partial u_{j}}{\partial x_{i}}-\frac{2}{3} \delta_{i j} \frac{\partial u_{k}}{\partial x_{k}}\right)\right]+\frac{\partial}{\partial x_{j}}\left(-\rho \overline{u_{\imath}^{\prime} u_{\jmath}^{\prime}}\right)
\end{gathered}
$$

Averaging procedure generate additional unknowns need to be solved and required the turbulence model flow problem closure. The last term in eq. (4) is a second-order tensor, derivative with respect to $x_{j}$, it defines the turbulence effect, and it's known as the Reynolds stress. To determine the value of the Reynolds stress [19], the $k-\varepsilon$ model was used.

Set of eq. (3)-(4) with $k$ - $\varepsilon$ turbulence model consists of 6 equations that need to be discretised and solved in order to obtain a solution for the velocities and pressure. In this work Ansys Fluent was used as a fluid flow solver. To produce a reliable model to study the flow generated by the novel impeller, the simulation results were compared with the results for a Rushton Turbine obtained in literature [16]. For the validation analysis, the same as in literature conditions were used: water as a working fluid with a viscosity of $\mu=0.001003 \mathrm{~kg} /(\mathrm{m} \cdot \mathrm{s})$ and a density of $\rho=998.2 \mathrm{~kg} / \mathrm{m}^{3}$. The vessel diameter $D$ was, $27 \mathrm{~cm}$, and equal to the vessel height $H(D=H)$. The impeller was mounted centrally and a disc diameter was $9.3 \mathrm{~cm}$.
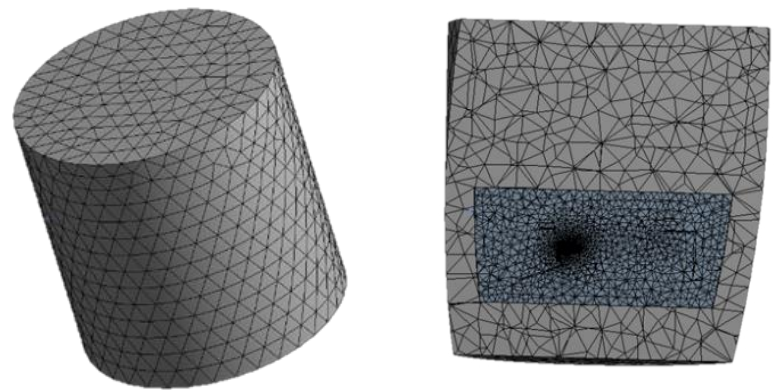

Fig. 2. Mesh of the computational domain for CFD simulation outside (left) and inside (right) the domain.

The impeller speed was variable and the Reynolds number calculated from eq. (5) was in the range from $R e=1$ 100000. Computational grid was tested for various grids, and the final resolution consist of 190000 control volumes (Fig. 2).

\section{Results and discussion}

As every industrial process, the main target has ever been to find an efficient way of mixing using as low power as possible. Figure 3 shows the Power number $N_{p}$ (eq. 5), calculated for various Reynolds numbers for Rushton turbine and Novel impeller, and compared with the experimental data from the literature for Rushton turbine [16]. As can be seen, the prediction for the Rushton turbine agrees relatively well with numerical results. Power number for the novel impeller is much lower and depend on the Reynolds number, power consumption is about 10 times lower for novel impeller than for the Rushton turbine. Power number depends on flow regime and for turbulence regime ( $\operatorname{Re}$ above 750$), N_{p}$ remains constant.

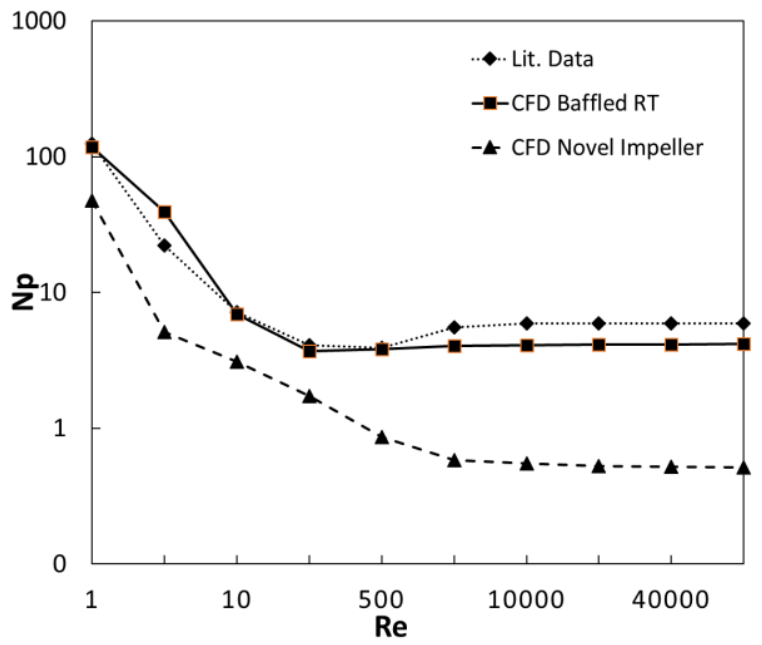

Fig. 3. Comparison of power number $N_{p}$ of CFD predictions with literature data [16].

The Power number and Reynolds number are calculated according to the equations:

$$
N_{P}=\frac{P}{\rho N^{3} D^{5}} \quad ; \quad R e=\frac{N D^{2} \rho}{\mu}
$$

where $\rho$ is the density, $\mu$ is the viscosity, $N$ is the number of rotation per minute, $D$ is the diameter and $P$ is the actual power that depends on the torque and can be evaluated as follows:

$$
P=2 \pi N \tau
$$

where $\tau$ is the torque.

Given the lower Power number $N_{p}$ of the novel impeller, it's worth to keep track of the pumping capacity, which is also different from the one of the Rushton Turbine. Figure 4 shows the radial pumping capacities for different Reynolds number through a cylindrical surface with a radius of $0.05 \mathrm{~m}$. In eq. (7), radial pumping capacity 
is obtained by integrating the mean radial velocities over the whole axial positions, i.e. from $z_{1}=0 \mathrm{~m}$ to $z_{2}=$ $0.27 \mathrm{~m}$.

$$
Q_{r}=2 \pi r \int_{z_{1}}^{z_{2}}\left|u_{r}\right| d z
$$

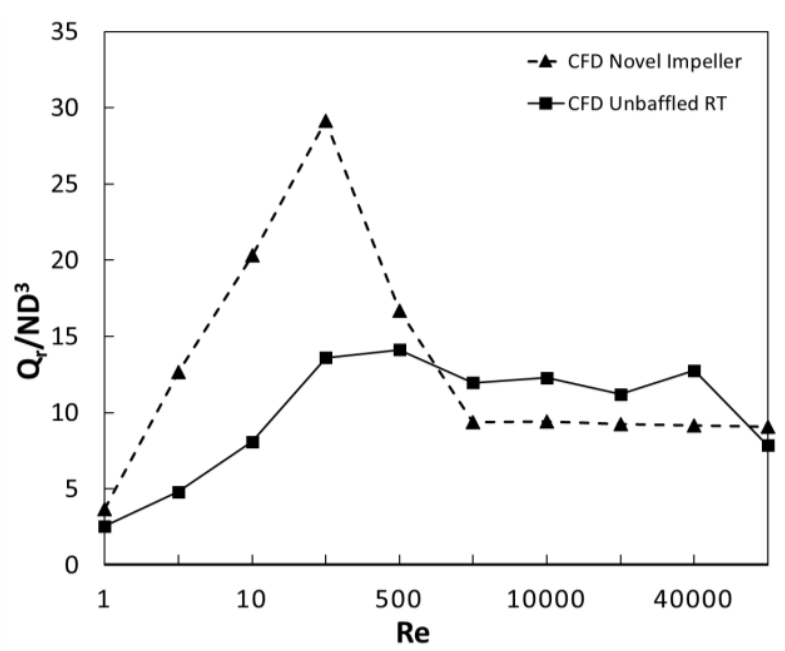

Fig. 4. Non-dimensional radial pumping capacity $Q_{r}$ in function of Reynolds number.

Figure 5 shown the velocity magnitude contour in $x-y$ cross-section plane for $\mathrm{z}=7 \mathrm{~cm}$ and in $\mathrm{y}-\mathrm{z}$ cross-section plane for $\varphi=0^{\circ}$ and for rotational speed $N=200 \mathrm{rpm}$. One may infer from this figure high-velocity magnitude in the internal part of the impeller jet type blades.

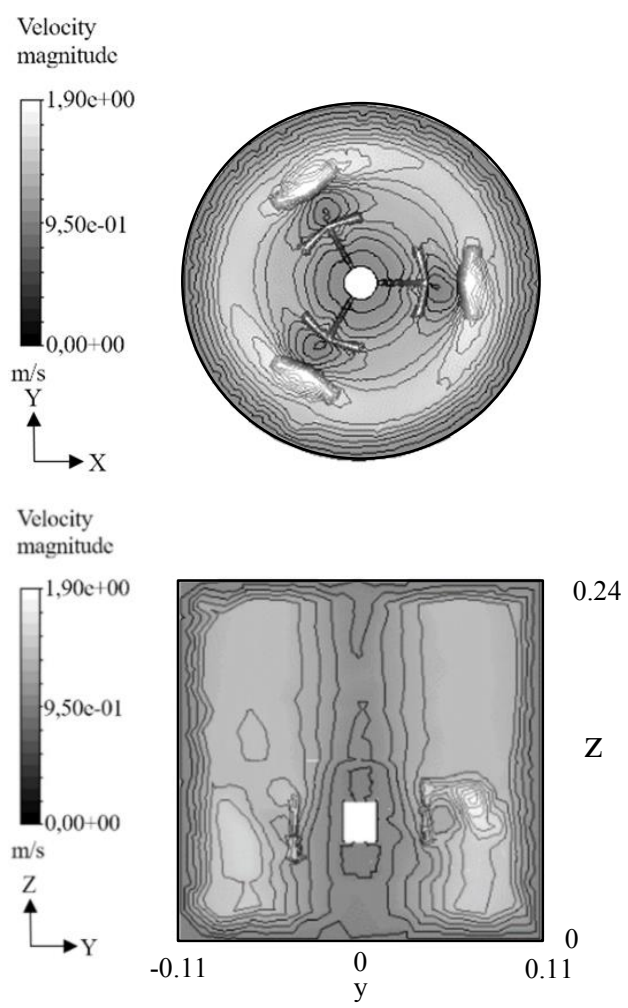

Fig. 5. Velocity magnitude contour in $x-y$ (top) and $y-z$ (bottom) cross-section plane and for $N=200 \mathrm{rpm}$.
In Figure 6 velocity vectors in $x-y$ cross-section plane for $\mathrm{z}=7 \mathrm{~cm}$ and in $\mathrm{y}-\mathrm{z}$ cross-section plane for $\varphi=0^{\circ}$ and for rotational speed $N=200 \mathrm{rpm}$ are presented. It can be seen in a height angular fluid motion as well as significant radial and axial flow effect for novel impeller. Flow field obtained from the numerical analysis has been compared to the experimental results (Fig.7.) obtained using Particle Image Velocimetry method.
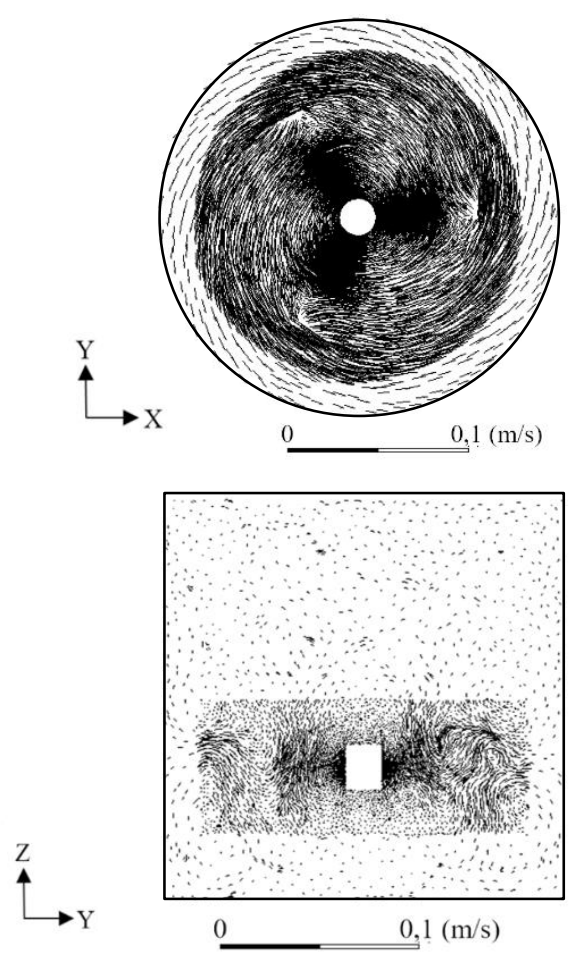

Fig. 6. Velocity vector in $\mathrm{x}-\mathrm{y}$ (top) and $\mathrm{y}-\mathrm{z}$ (bottom) crosssection plane and for $N=200 \mathrm{rpm}$.
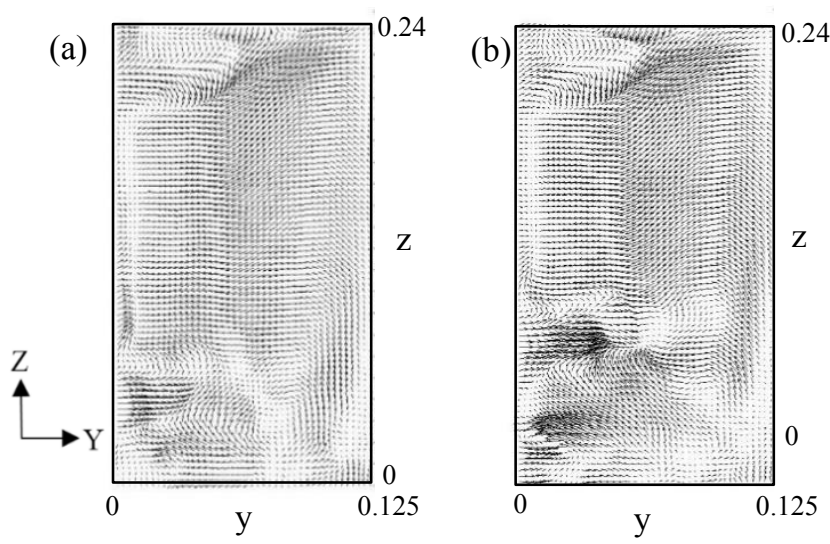

Fig. 7. Velocity field from experimental measurement for $N=140 \mathrm{rpm}$ with the impeller at two different heights: $h=3 \mathrm{~cm}$ (a) and $h=6 \mathrm{~cm} \mathrm{(b).}$

\section{Conclusions}

Using the proposed impeller type, it is possible to reduce the power number $N_{p}$ for low as well high Reynolds number. After exceeding the critical for the phenomena value for the Reynolds number about $R e=750$, turbulent regime occurs, and power number stabilization is 
observed. The power number values for the classic Rushton Turbine are much higher, and the stabilization of $N_{p}$ values is observed for a smaller Reynold number. The Power number is at least 6-10 times higher for the Rushton Turbine then for the novel impeller. This results in higher power consumption at the same number of revolutions. The tested new impeller type is a promising alternative to the classic rotating disc or other blades and non-blades based impeller types. It is worth to notice that pumping number for proposed impeller depends on Reynolds number is still much higher or a bit lower than the pumping number $Q_{r}$ generate by the Rushton turbine. This also means that efficiency which can be defined as a ratio of pumping number $\mathrm{Q}_{\mathrm{r}}$ to the power number $N_{p}$ for the novel impeller is significantly higher than calculated for reference ones.

ACKNOWLEDGMENTS. The present work was partially supported by the Polish Ministry of Science (Grant MNiSW/2019/162/DIR)

\section{References}

1. M. Jaszczur, A. Młynarczykowska, R. Hanus, J. of Physics. Conf. Series, ISSN 1742-6588, 745032121 (2016)

2. F. Scargiali, A. Busciglio, F. Grisafi, A. Tamburini, G. Micale Ind. Eng. Chem. Res. 52 (2013)

3. T. Su, F. Yang, M. Li, K. Wu, Chinese J. of Chem. Eng. 26 (2017)

4. F. Scargiali, A. Tamburini, G. Caputo, G. Micale, Chem. Eng. Res Des. 132 (2017)

5. T. Kumaresan, B. Jyeshtharaj, B. Joshi, Chem. Eng. J. 115 (2005)

6. M. Basavarajappa, T. Draper, P. Toth, T. A. Ring, S. Miskovic, Minerals Engineering, 83 (2015)

7. H. Ameur, Y. Kamla, D. Sahel, Per. Pol. Mech. Eng. 62, 4 (2018)

8. M. Major-Godlewska, J. Karcz, Chem Zvesti. 72, 5 (2018)

9. J. Karcz, B. Mackiewicz, Chem, Papers. 63, 2 (2009)

10. J. Karcz, M. Major, Chem. Eng. Proc. 37 (1998)

11. A. Delafosse, A. Line, J. Morchain, P. Guiraud, Chem. Eng. Res. Design, 86 (2008)

12. A. Delafosse, M.L. Collignon, S. Calvo, F. Delvign, M. Crine, P. Thonart, D. Toye, Chem. Eng. 106 (2013)

13. M. Jaszczur, Archives of Mechanics, 8 (2011)

14. M. Jaszczur, L.M. Portela, ERCOFTAC 12 (2008)

15. J. Derksen, H.E.A. van den Akker, AIChE J. 45, 2 (1999)

16. S. Malika, E. Lévêquea, L. Bouaifi, L. Gamet, E. Flottes, S. Simoëns, M. El-Hajem, Chem. Eng. Res Design, 108 (2016)

17. H. Patil, A.K. Patel, H.J. Pant, A.V. Vinod, ISH J of Hydr. Eng. 2, 2 (2018)
18. J. H. Rushton, E.W. Costich, H.J. Everett, Chem. Eng. Progress, 46 (1950)

19. H, Singh, D.F. Fletcher, J.J. Nijdam, Chem. Eng. Sci. 66 (2011)

20. B. N. Murthy, J. B. Joshi, Chemica Engineering Science, 63 (2008)

21. P. Mavros, Inst. of Chem. Eng. 79 (2001) 